Check for updates

Cite this: Mater. Adv., 2021, 2, 6631

Received 19th March 2021 ,

Accepted 26th August 2021

DOI: 10.1039/d1ma00239b

rsc.li/materials-advances

\title{
A first-principles study of the relationship between modulus and ideal strength of single-layer, transition metal dichalcogenides
}

\author{
Hao Sun, ${ }^{a}$ Pratyaksh Agrawal $^{a}$ and Chandra Veer Singh (D) *ab
}

\begin{abstract}
Electronic properties of single-layer transition metal dichalcogenides (TMDs), such as bond gap, can be tuned by elastic strain. The regulating range of such strain engineering is determined by ideal strengths, which, according to Griffth's strength limit, is usually estimated as $E / 10$, where $E$ is the elastic modulus. Despite being extensively used, this relationship between ideal strength and moduli has yet to be thoroughly investigated for TMDs. Our extensive density functional theory calculations on six representative, single-layer TMDs $\left(\mathrm{MoS}_{2}, \mathrm{MoSe}_{2}, \mathrm{NbS}_{2}, \mathrm{NbSe}_{2}, \mathrm{ReS}_{2}, \mathrm{ReSe}\right.$ ) showed that the moduli of TMDs increase as their transition metal elements change from the $V$ to VII group. However, despite having higher moduli, $\mathrm{ReS}_{2}$ and $\mathrm{ReSe}_{2}$ exhibit lower strengths and failure strains than $\mathrm{MoS}_{2}, \mathrm{MoSe}_{2}$, and $\mathrm{NbSe}_{2}$. Such strength degeneration is attributed to the multiple bond directions in $\mathrm{ReS}_{2}$ and $\mathrm{ReSe}_{2}$. As strain softening renders stretched bonds easier to deform, deformation gradually concentrates on the bonds most close to the loading direction. Since only a small portion of covalent bonds are stretched, the ideal strength of the whole structure is diminished. Overall, our findings suggest that reducing the variety of bond orientations could increase the apparent ductility of TMDs without decreasing the strength.
\end{abstract}

\section{Introduction}

Monolayer transition metal dichalcogenides (TMDs) have attracted intensive attention due to their unique chemical and physical properties. Unlike graphene, whose zero band gap leads to a low intrinsic on-off current ratio, ${ }^{1}$ the direct band gap (1.1-2.0 eV $)^{2-4}$ of TMDs significantly enhances the on-off current ratio, ${ }^{5}$ the luminescence quantum efficiency, ${ }^{6-8}$ and the intrinsic electronic mobility. ${ }^{9}$ These outstanding characteristics render TMDs interesting for both fundamental research and applications in field-effect transistors, ${ }^{5}$ phototransistors, ${ }^{10}$ optoelectronics, ${ }^{11}$ and mechanical resonators. ${ }^{12}$

The advantages of TMDs mentioned above have stimulated extensive research on their electronic band structures. Both experiments ${ }^{11}$ and first-principles calculations ${ }^{13-17}$ have found that elastic strain can effectively tune the band gap of TMDs. The adjustment range of band gap is determined by the maximum strain a TMD can sustain. Theoretically, a large enough tensile strain can even tune the conduction band

\footnotetext{
${ }^{a}$ Department of Mechanical and Industrial Engineering, University of Toronto, Toronto, ON, M5S 3G8, Canada. E-mail: chandraveer.singh@utoronto.ca; Tel: +1416 946-5211

${ }^{b}$ Department of Materials Science and Engineering, University of Toronto, Toronto, ON, M5S 3E4, Canada
}

minimum to be lower than the Fermi level, resulting in a metal-like TMD. ${ }^{13}$ The critical strain corresponding to such transition was found to be related to the moduli of $\operatorname{TMDs}^{18}$ and scale roughly with its ideal strength. ${ }^{19}$ Hence, strain engineering can effectively adjust the electronic band structure of TMDs without varying chemical components and topological atomic arrangements.

As the modulus and ideal strength are two key factors determining the limit of strain engineering, robust literature has investigated the mechanical property of $\mathrm{MoS}_{2}{ }^{12,20-26}$ and compared its properties with other TMDs. ${ }^{27-30}$ The bending stiffness of TMDs was found to increase as the transition metal goes from the IV to VI group. ${ }^{31}$ The $2 \mathrm{D}$ elastic stiffness of TMDs was reported to depend on the bond lengths ${ }^{28}$ and to soften with increasing lattice parameters. ${ }^{32,33}$ Despite such a significant expansion of knowledge, prior analysis has only focused on linear mechanical properties..$^{27,28,30,32,33}$ The nonlinear mechanical properties of TMDs, such as ideal strengths and fracture strains, have yet to be thoroughly investigated.

Without a complete structure-mechanical-property relationship, the ideal strength of TMDs can only be estimated by Griffith's strength limit-a linear correlation between the ideal strength and Young's modulus. ${ }^{29}$ This relationship, however, is based on a hypothesis that the deformation is mainly undertaken by bond stretching, ignoring the role of bond rotation. Recent research on 
graphene allotropes has found that bond rotation can significantly lessen bond stretch, enhancing fracture strain. ${ }^{34,35}$ In one graphene allotrope with a Poisson's ratio as high as 0.8 , bond rotation leads to an S-shape stress-strain curve, akin to that of elastomers, with an ultimate tensile strength (UTS) exceeding the Griffth's cohesive strength limit. ${ }^{34}$ Despite the intense study on graphene allotropes, the bond-rotation effect on the mechanical properties of TMDs remains unexplored. Thus, a complete picture of the structuremechanical-property relationship for TMDs is still missing.

This paper presents a comprehensive theoretical analysis of the structure-mechanical-property relationship of six representative TMDs $\left(\mathrm{MoS}_{2}, \mathrm{MoSe}_{2}, \mathrm{NbS}_{2}, \mathrm{NbSe}_{2}, \mathrm{ReS}_{2}, \mathrm{ReSe}_{2}\right)$ with varying chemical components and topological atomic arrangements. For most of the six TMDs, their nonlinear mechanical properties, such as ideal strengths and fracture strains, have not been reported elsewhere. The relationship between the UTS and modulus was investigated from two perspectives. First, the physical insight of Griffth's strength limit in TMDs was revealed based on the charge-density evolution of their covalent bonds during bond stretching; then we analyzed the effect of bond rotation on the mechanical properties of the TMD. Finally, different fracture mechanisms of TMDs were discussed. The fundamental insights revealed by this research can help experimentalists analyze the fracture mechanism of TMDs.

\section{Methodology}

The atomic configurations of the six TMDs are shown in Fig. 1. All DFT simulations were performed using the Quantum-ESPRESSO package, ${ }^{36}$ pseudopotentials with the Perdew-Berke-Ernzerhof (PBE) exchange-correlation functional, generalized gradient approximation, ${ }^{37}$ and a $13 \times 13 \times 3 \mathrm{k}$-point Monkhorst-Pack grid. ${ }^{38}$ The kinetic energy cut-offs of 60 and 480 Ry were used for the wavefunctions and charge density, respectively. The convergence criterion of the self-consistent field (SCF) procedure was set to $1.0 \times 10^{-6} \mathrm{Ry}$. A 20-angstromthick vacuum in the out-of-plane direction was used to avoid any interlayer interactions. Each system was initially relaxed until the magnitude of the residual Hellmann-Feynman force on each atom was less than 0.001 Ry per bohr.

To simulate the uniaxial and biaxial tension, the unit cells illustrated in Fig. 1 were subjected to different magnitudes of uniaxial or equal-biaxial strains (see Fig. 1 for cell orientation). Strains were applied by dilating the unit cells along the $x$ or $y$ directions and then applying an equal affine transformation to all atomic positions. The deformed unit cell was then subjected to an energy minimization routine to obtain its ground state configuration under the given boundary conditions. The UTS of the resultant stress-strain curve is the ideal strength of the TMD, and the corresponding strain is the fracture strain. To obtain stress values in $2 \mathrm{D}$ terms with units $\mathrm{N} / \mathrm{m}$, we multiply the UTS by the thickness of each TMD, which is the thickness of each monolayer TMD structure plus the gap between two TMD layers $\left(\sim 3 \AA^{39}\right)$ determined by van der Waals interaction.

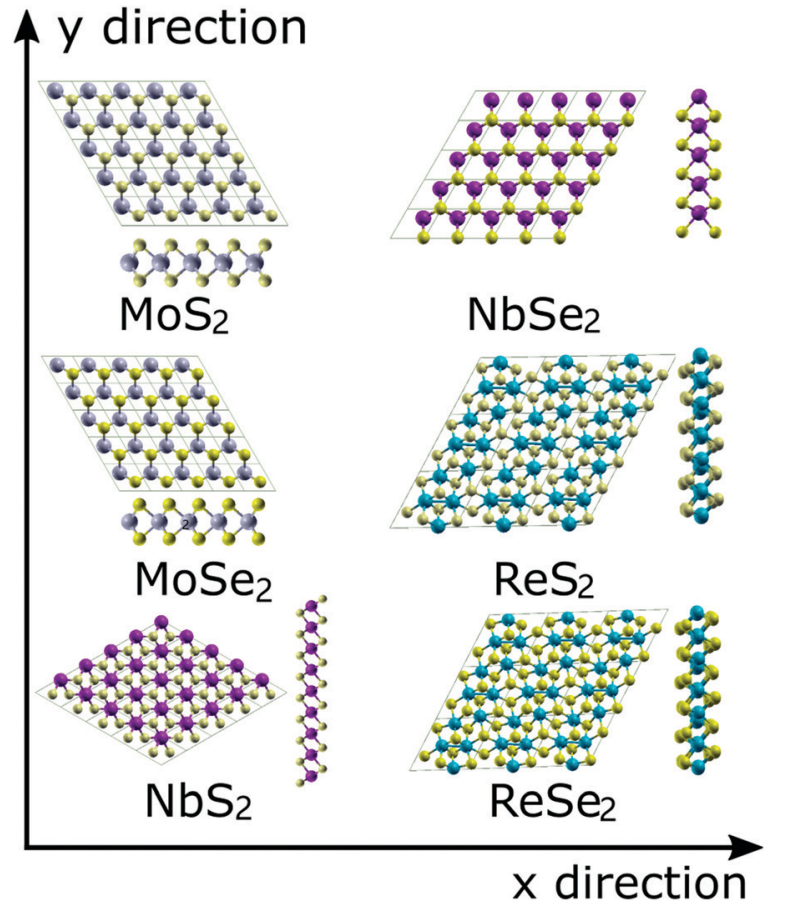

Fig. 1 Atomic topologies and the unit cells for the six examined TMDs. The two defomation directions ( $x$ and $y$ ) are also illustrated by arrows.

\section{Results and discussion}

\subsection{Stress-strain responses}

The knowledge of mechanical properties not only provides physical insights into the nature of covalent bond interactions in TMD monolayers, but is also essential for practical applications of TMDs in modern technology. The true stress versus engineering strain responses for the six TMDs are shown in Fig. 2. At strains smaller than approximately 5\%, a linear stress-strain relationship is valid for all TMDs. At strains larger than 5\%, all stress-strain curves become nonlinear. For TMDs such as $\mathrm{MoS}_{2}$ and $\mathrm{MoSe}_{2}$, strain softening levels off the stress-strain curve gradually until the UTS point, after which mechanical instability occurs. However, $\mathrm{ReS}_{2}, \mathrm{ReSe}_{2}, \mathrm{NbS}_{2}$, and $\mathrm{NbSe}_{2}$ undergo brittle fracture with a sudden drop in stress magnitudes. $\mathrm{MoS}_{2}$ and $\mathrm{MoSe}_{2}$ exhibit higher UTS and larger failure strain than $\mathrm{ReS}_{2}$ and $\mathrm{ReSe}_{2}$, combining both high strength and high facture strain.

Linear mechanical properties, such as elastic constants $C_{11}$, $C_{22}, C_{12}$, and $C_{66}$, are readily calculated from the initial slopes of different stress-strain responses of TMDs. Since $C_{22}$ is approximately equal to $C_{11}$, the $2 \mathrm{D}$ layer modulus, a quantity that represents the resistance of a nanosheet to stretching, can be calculated as ${ }^{40}$

$$
\gamma_{2 \mathrm{D}}=\frac{1}{2}\left(C_{11}+C_{12}\right)
$$

Other linear mechanical properties, such as Young's modulus $(E)$, Poisson's ratio $(\nu)$, and shear modulus $(G)$, can be 

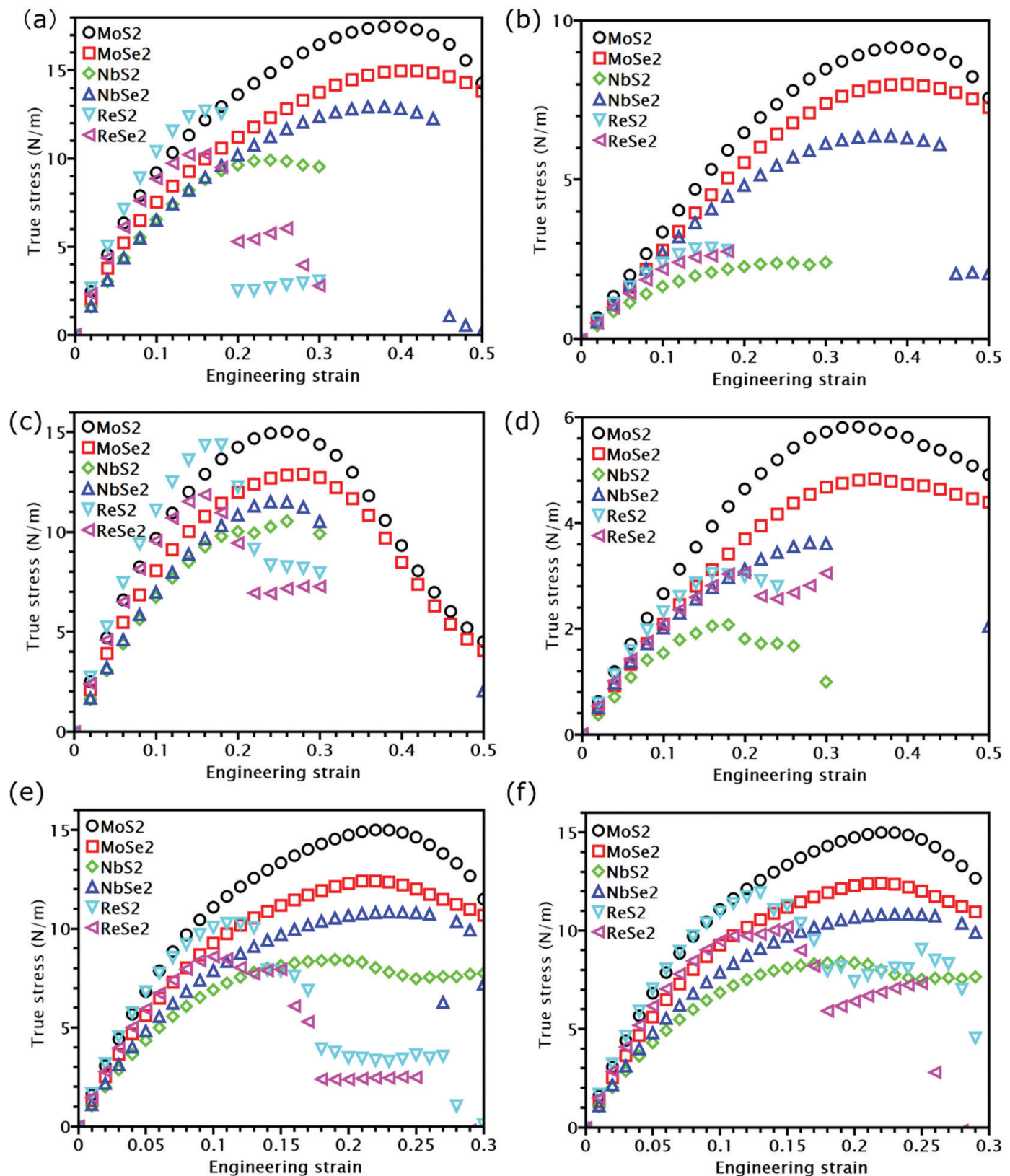

Fig. 2 Stress-strain curves for all six examined TMDs. (a) and (b) represent the stress-strain responses under $x$-uniaxial tension along the $x$ and $y$ directions, respectively; (c) and (d) exhibit the stress-strain curves under $y$-uniaxial straining in the $x$ and $y$ directions, respectively; (e) and (f) show the stress-strain responses in biaxial tension along the $x$ and $y$ directions, respectively.

obtained using the following expressions:

$$
E=\frac{C_{11^{2}}-C_{12}{ }^{2}}{C_{11}}, \quad v=\frac{C_{12}}{C_{11}}, \quad G=C_{66}
$$

All calculated linear mechanical properties for the six TMDs are listed in Table 1. Our data are in excellent agreement with the reported values in the literature from theoretical and experimental studies. ${ }^{41,42}$ Compared with graphene, ${ }^{43}$ all TMDs have smaller elastic constants, Young's modulus, shear modulus, and 2D layer modulus, indicating weaker covalent bonds in TMDs. Although the elements in TMDs have more protons in their nuclei than carbon atoms, the larger atomic radii and stronger shielding effect due to more inner electrons diminish the attraction between the outermost electrons and the nuclei. This weakening effect can be quantified by the first ionization energies of different elements, which have the following order: $\mathrm{C}\left(1086 \mathrm{~kJ} \mathrm{~mol}^{-1}\right)>\mathrm{S}\left(999 \mathrm{~kJ} \mathrm{~mol}^{-1}\right)>$ $\mathrm{Se}\left(941 \mathrm{~kJ} \mathrm{~mol}^{-1}\right)>\operatorname{Re}\left(760 \mathrm{~kJ} \mathrm{~mol}^{-1}\right)>\operatorname{Mo}\left(684 \mathrm{~kJ} \mathrm{~mol}^{-1}\right)>$ $\mathrm{Nb}\left(652 \mathrm{~kJ} \mathrm{~mol}^{-1}\right) .{ }^{44}$ This order explains the dependence of the Young's modulus of TMDs on their chemical components: given the same non-metallic elements, the Young's modulus of TMDs increases as the transition metal goes from the V to VII group (Fig. 3(c)); given the same metallic elements, the TMD containing $\mathrm{S}$ atoms has larger Young's modulus than that having Se atoms. 
Except in-plane deformation, we also investigated the bending modulus $(D)$ for a $2 \mathrm{D}$ nanosheet ${ }^{45}$

$$
D=\frac{E h^{2}}{12\left(1-v^{2}\right)}
$$

where $h$, the thickness of the TMD, is difficult to determine accurately because the electronic configuration along the normal direction changes during deformation. However, the lowest estimate of $D$ can be calculated using the absolute thickness of
As listed in Table 1, given the same length $L$, the critical buckling strain for TMDs can be ten times larger than that for graphene due to a larger $D$ and smaller $E$. Hence, compared to monolayer graphene, TMDs are more robust for in-plane structural deformations and are more resistant to buckling.

Next, we investigated the direction dependent Young modulus $E(\theta)$ and Poisson's ratio $\nu(\theta)$ along an arbitrary in-plane direction $\theta$ ( $\theta$ is the angle relative to the $x$ direction); both can be expressed by elastic constants as follows ${ }^{47}$

$$
\begin{gathered}
E(\theta)=\frac{C_{11} C_{22}-C_{12}{ }^{2}}{C_{11} \sin ^{4}(\theta)+C_{22} \cos ^{4}(\theta)+\left(\frac{C_{11} C_{22}-C_{12}{ }^{2}}{C_{66}}-2 C_{12}\right) \sin ^{2}(\theta) \cos ^{2}(\theta)} \\
\nu(\theta)=-\frac{\left(C_{11}+C_{22}-\frac{C_{11} C_{22}-C_{12}{ }^{2}}{C_{66}}\right) \sin ^{2}(\theta) \cos ^{2}(\theta)-C_{12}\left(\cos ^{4}(\theta)+\sin ^{4}(\theta)\right)}{C_{11} \sin ^{4}(\theta)+C_{22} \cos ^{4}(\theta)+\left(\frac{C_{11} C_{22}-C_{12}{ }^{2}}{C_{66}}-2 C_{12}\right) \sin ^{2}(\theta) \cos ^{2}(\theta)}
\end{gathered}
$$

the nanosheet: $0.6-0.8 \AA$ for graphene ${ }^{46}$ and $\sim 3.13 \AA$ for TMDs. Although TMDs have lower modulus than graphene, their bending moduli are larger than that of graphene because of their much larger thicknesses (Table 1). Such difference in thickness is attributed to the three-layer, atomic structure of TMDs, which offers more interaction terms restraining the bending motion.

Once knowing $D$ and $E$, we can study the buckling phenomenon and estimate the critical buckling strain $\left(\varepsilon_{\mathrm{c}}\right)$ using Euler's buckling theorem ${ }^{45}$

$$
\varepsilon_{\mathrm{c}}=-\frac{4 \pi^{2} D}{E L^{2}}
$$

Table 1 List of the DFT calculated mechanical properties for all TMDs, including elastic constants $\left(C_{11}, C_{22}\right.$, and $\left.C_{12}\right)$, UTS, fracture strain $(\varepsilon)$, Young's modulus $(E)$, Layer modulus $(\gamma)$, bending modulus $(D)$, critical bulking strain $\left(\varepsilon_{c}\right)$, and Poisson's ratio $(\nu)$. All moduli and strengths are in $N / m$ unit

\begin{tabular}{llllllll}
\hline & $\mathrm{MoS}_{2}$ & $\mathrm{MoSe}_{2}$ & $\mathrm{NbS}_{2}$ & $\mathrm{NbSe}_{2}$ & $\mathrm{ReS}_{2}$ & $\mathrm{ReSe}_{2}$ & Graphene $^{41}$ \\
\hline$C_{11}$ & 133.36 & 114.56 & 90.09 & 86.58 & 140.77 & 120.55 & 359 \\
$C_{12}$ & 37.05 & 31.89 & 25.61 & 30.74 & 31.12 & 26.55 & 65.1 \\
$\mathrm{UTS}_{x}$ & 17.48 & 14.97 & 9.91 & 12.95 & 12.7 & 10.28 & 31.2 \\
$\varepsilon_{x}$ & 0.39 & 0.41 & 0.23 & 0.37 & 0.16 & 0.15 & 0.23 \\
$C_{22}$ & 134.26 & 114.5 & 90.03 & 89.53 & 142.11 & 125.17 & 359 \\
$C_{21}$ & 36.15 & 30.59 & 22.12 & 28.47 & 31.74 & 25.61 & 65.1 \\
$\mathrm{UTS}_{y}$ & 15.01 & 12.89 & 10.67 & 11.52 & 14.45 & 11.85 & 29.3 \\
$\varepsilon_{y}$ & 0.26 & 0.27 & 0.23 & 0.25 & 0.17 & 0.16 & 0.18 \\
$\mathrm{UTS}_{\text {biax }}^{x}$ & 15.00 & 12.42 & 8.43 & 10.83 & 10.26 & 8.59 & 33.2 \\
$E_{\text {biax }}^{x}$ & 160.57 & 135.04 & 108.74 & 114.71 & 167.48 & 144.39 & 418 \\
$\mathrm{UTS}_{\text {biax }}$ & 15 & 12.41 & 8.41 & 10.83 & 11.93 & 15.67 & 33.2 \\
$E_{\text {biax }}^{v}$ & 160.54 & 134.98 & 111.79 & 116.11 & 170.58 & 151.37 & 418 \\
$\varepsilon_{\text {biax }}$ & 0.23 & 0.22 & 0.19 & 0.25 & 0.12 & 0.09 & 0.23 \\
$G$ & 48.6 & 41.13 & 18.65 & 28.08 & 59.42 & 53.53 & 147 \\
$\gamma_{2 \mathrm{D}}$ & 85.21 & 73.23 & 57.85 & 58.66 & 85.95 & 73.55 & 212 \\
$E$ & 123.07 & 105.68 & 82.81 & 75.67 & 133.89 & 114.70 & 347 \\
$\nu$ & 0.28 & 0.28 & 0.28 & 0.36 & 0.22 & 0.22 & 0.18 \\
$D(\mathrm{eV})$ & 6.8 & 5.84 & 4.59 & 4.41 & 7.17 & 6.14 & 1.2 \\
$\varepsilon_{\mathrm{c}} \cdot L^{2}$ & 34.94 & 34.95 & 35.06 & 36.86 & 33.87 & 33.86 & 2.2
\end{tabular}

As shown in Fig. 3(a) and (b), TMDs with hexagonal structures, such as $\mathrm{MoS}_{2}, \mathrm{MoSe}_{2}$ and $\mathrm{NbSe}_{2}$, are approximately isotropic: their $E(\theta)$ and $\nu(\theta)$ are independent of angular variation. $\operatorname{ReS}_{2}$ and $\mathrm{ReSe}_{2}$ exhibit slight anisotropic behaviors: their $E(\theta)$ peaks are at an angle of 45 degrees with respect to the $x$-uniaxial loading direction. The $\theta$ corresponding to the largest modulus also shows the smallest Poisson's ratio. $\mathrm{NbS}_{2}$ shows the strongest anisotropic behavior; its $E(\theta)$ reaches the maximum in $x$ and $y$ directions, where its Poisson's ratio reaches minimum.

At last, we studied nonlinear mechanical properties such as UTS and failure strain for the six examined TMDs under different loading methods. Compared with graphene, ${ }^{43}$ all TMDs have lower UTS, but some TMDs show higher failure strain. Specifically, the failure strain of $\mathrm{MoS}_{2}$ and $\mathrm{MoSe}_{2}$ could even reach 0.4 in uniaxial tension. Nonetheless, previous research found that $\mathrm{MoS}_{2}$ can fracture before reaching the UTS point due to phonon instability. ${ }^{26}$ The actual fracture strength corresponding to phonon instability, however, is still close to the apparent $\mathrm{UTS}^{26}$ because the slope of the stressstrain curves is close to zero near the UTS point. Thus, the UTS listed in Table 1 can still be utilized as an approximate fracture strength even when phonon instability is considered. Due to their importance in strain engineering, the UTS and its relationship with the modulus of the six examined TMDs will be discussed in detail in the following section.

\subsection{The Griffth's strength limit of TMDs}

Instead of directly obtaining UTS from stress-strain curves, we can also estimate the ideal strength of TMDs as $E / 10$, where $E$ is the Young's modulus. ${ }^{29}$ Such an approximation of UTS is known as Griffth's strength limit. Despite being widely used to estimate the ideal strength of brittle materials, this limit is not suitable for all TMDs. For example, for TMDs with the same non-metallic elements, their moduli were found to increase as 
(a)
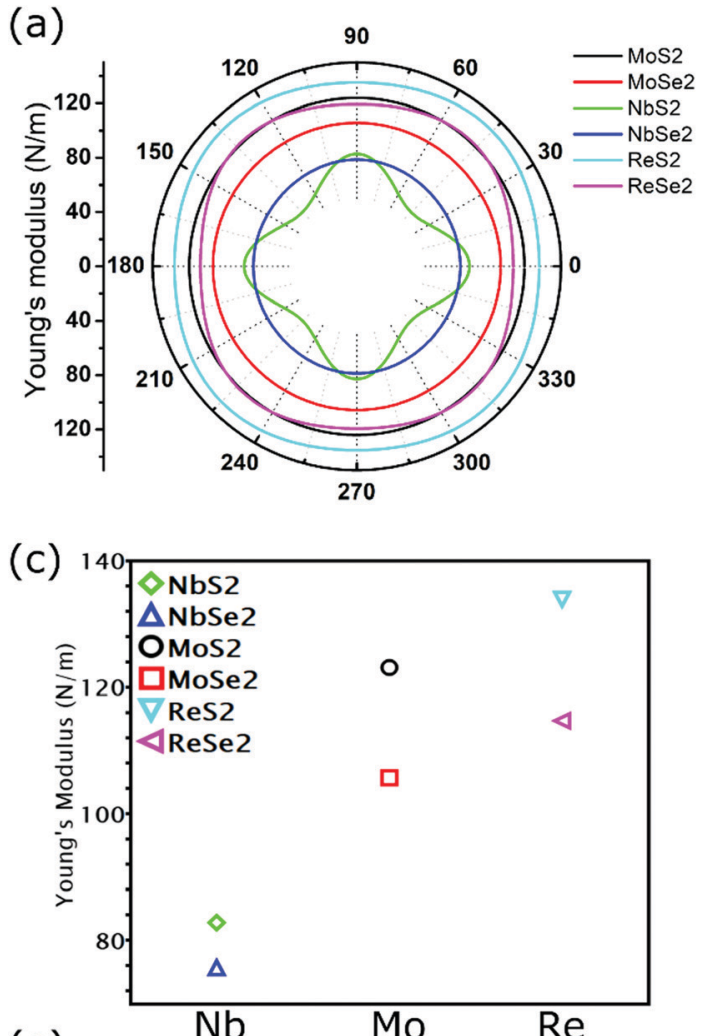

(e)

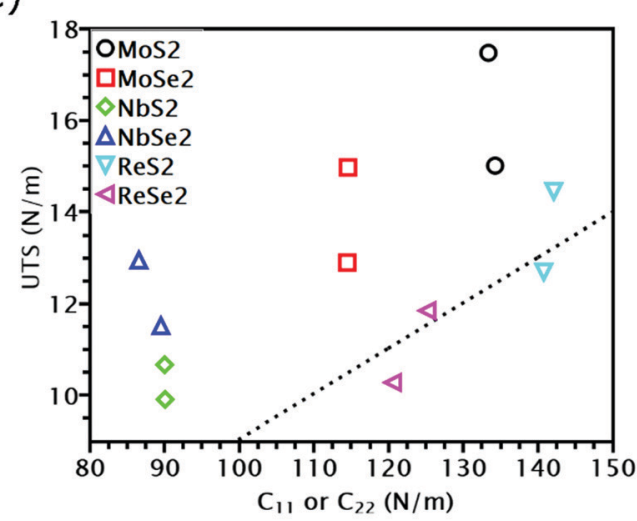

(b)

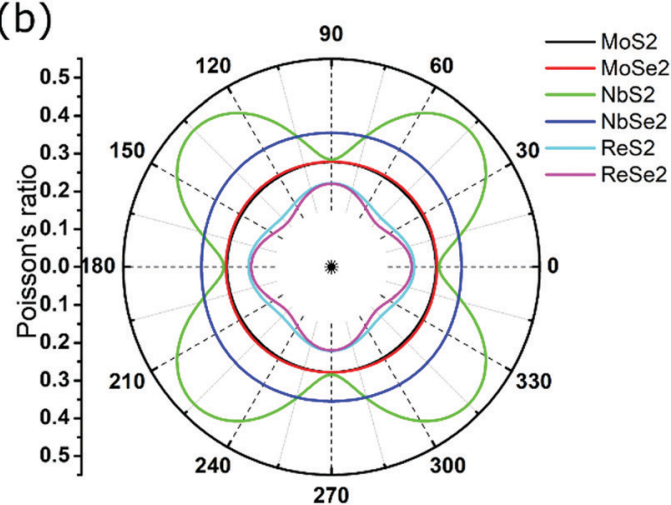

(d)

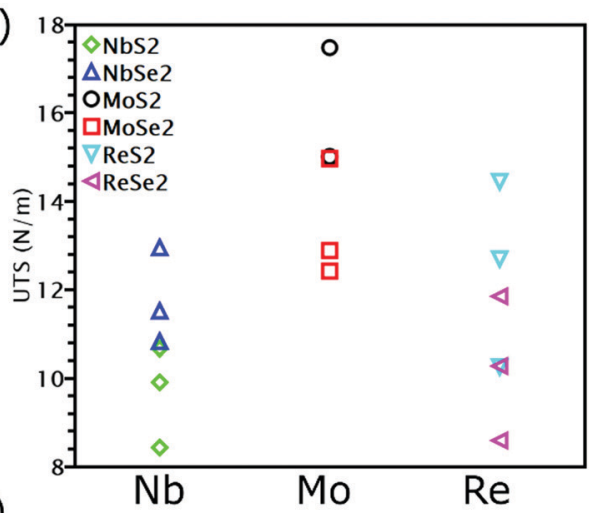

(f)

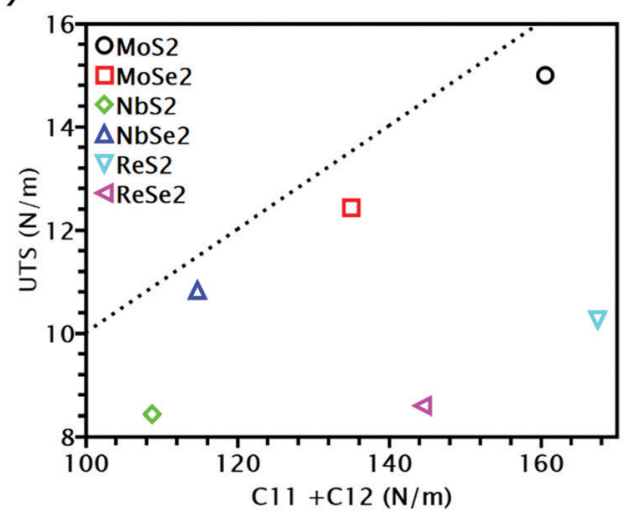

Fig. 3 The angular dependence of Young's modulus (a) and Poisson's ratio (b) for six TMDs. The modulus and UTS for different TMDs are illustrated based on their metallic elements in subfigure (c) and (d), respectively. (e) The relationship between UTS and $C_{11}$ in both $x$-uniaxial and $y$-uniaxial tension. (f) The relationship between UTS and $C_{11}+C_{12}$ in biaxial tension. The dashed lines in (e) and (f) represent the relationship between UTS and elastic constants predicted by the Griffth's strength limit.

the transition metal goes from the V to VII group (Fig. 3(c)). However, $\mathrm{MoS}_{2}$ and $\mathrm{MoSe}_{2}$, despite having smaller Young's modulus than $\mathrm{ReS}_{2}$ and $\mathrm{ReSe}_{2}$, exhibit higher UTS (Fig. 3(d)). Thus, E/UTS for $\mathrm{ReS}_{2}$ and $\mathrm{ReSe}_{2}$ is around 11, while that for $\mathrm{MoS}_{2}$ and $\mathrm{MoSe}_{2}$ is approximately 7 . These results indicate that it could be possible for TMDs to overcome the Griffth's strength limit.

However, our previous research ${ }^{34}$ has found that Young's modulus equals the slope of the stress-strain curve only in uniaxial tension with a free-boundary condition in the lateral direction, while in our DFT simulations, a fixed boundary condition is applied. Thus, instead of relating UTS with
Young's modulus, we plotted the UTS in uniaxial tension with $C_{11}$ and UTS in biaxial tension with $C_{11}+C_{12}$ in Fig. 3(e) and (f), respectively. As shown in Fig. 3(e), except for two uniaxial tensions of $\mathrm{ReS}_{2}$ and $\mathrm{ReSe}_{2}$, the UTS of all TMDs is above the dash line corresponding to $C_{11} / \mathrm{UTS}=10$, indicating that the UTS of most TMDs is larger than $C_{11} / 10$. However, for biaxial tension, the UTS of all TMDs is below the dash line, with values smaller than $\left(C_{11}+C_{12}\right) / 10$ (Fig. 3(f)). Previous research has found that the reduction of the ratio between modulus and UTS is attributed to less contribution from bond rotation in biaxial tension, ${ }^{34}$ but these work only focus on graphene allotropes, while the influence of chemical components could also be 
essential in TMDs. In the next section, we will discuss the mechanical properties of covalent bonds formed by different elements.

\subsection{Mechanical properties of covalent bonds in TMDs}

As discussed above, the influence of chemical components on the mechanical properties of different covalent bonds in TMDs remain unexplored. It is possible that some TMDs may be composed of covalent bonds whose strengths $S$ deviates from
$K / 10$, where $K$ is the bond stiffness. As a result, the TMD composed of such weaker bonds could have UTS smaller than $E / 10$. To verify this assumption, we applied volumetric expansion to the unit cell of all the six examined TMDs, so only bond stretch is allowed to accommodate the deformation. The distance between all atoms was equally increased while all bond angles remained unchanged. The corresponding energy increase per atom, $e(\varepsilon)$, obtained by self-consistent field (SCF) calculations in DFT, was only due to bond stretch
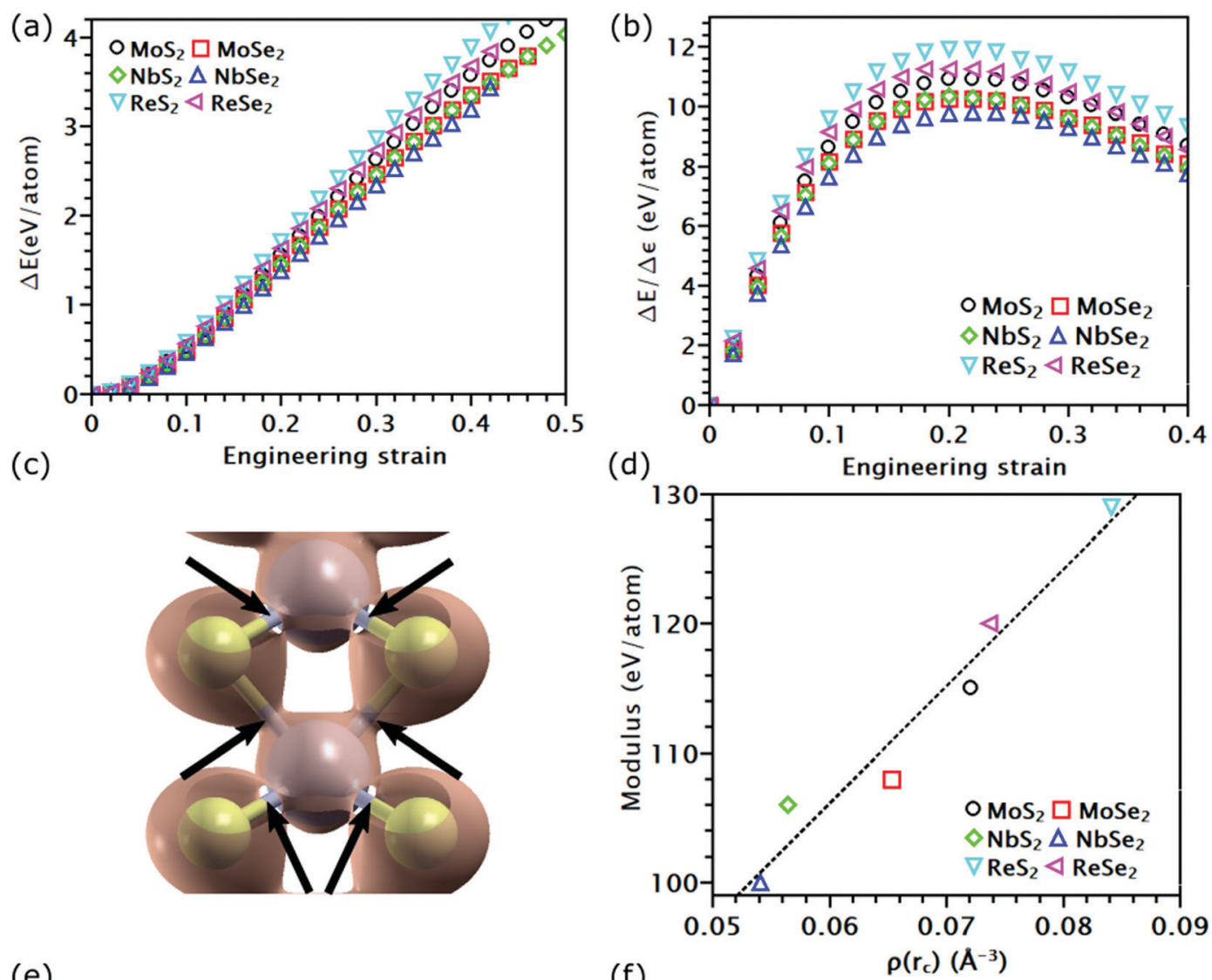

(e)
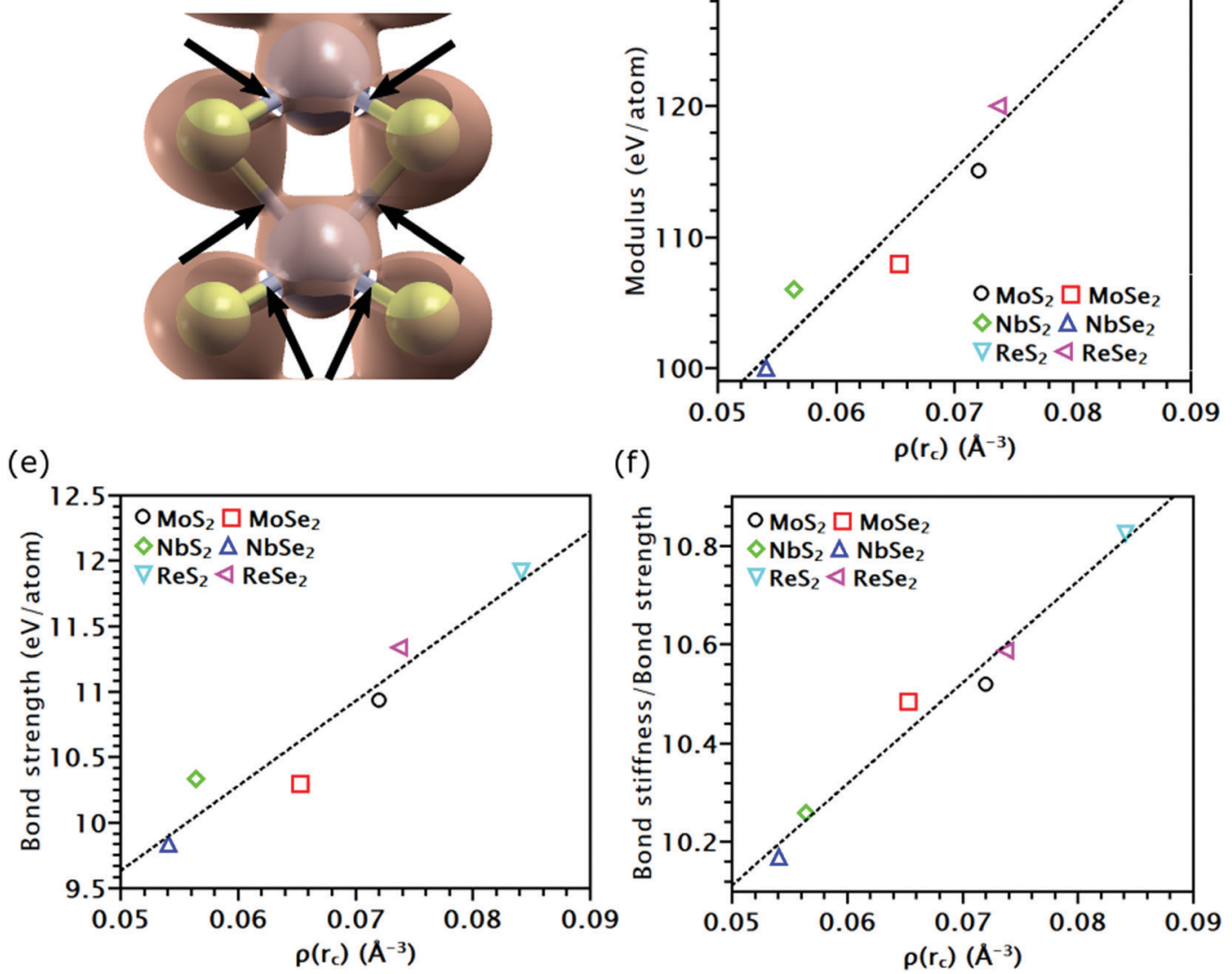

Fig. 4 A universal relationship between the charge-density evolution and mechanical properties of all covalent bonds in the examined TMDs. (a) The potential energy increase of the six examined TMDs as a function of the volumetric strain. (b) The bond-force-bond-strain response of the six kinds of covalent bonds in TMDs. (c) A charge-density iso-surface in $\mathrm{MoS}_{2}$ with a value of $0.065 \AA^{-3}$. The bond critical points, $r_{C}$, are marked by black arrows. (d) The relationship between $\rho\left(r_{\mathrm{C}}\right)$ and bond stiffness $K$. (e) The relationship between $\rho\left(r_{\mathrm{C}}\right)$ and bond strength $S$. (f) The relationship between $\rho\left(r_{\mathrm{C}}\right)$ and $K / S$. 
(Fig. 4(a)). The force required to stretch all covalent bonds was calculated as the increasing rate of $e(\varepsilon)$ with the applied strain, $P(\varepsilon)=\mathrm{d} e(\varepsilon) / \mathrm{d} \varepsilon$.

Like the stress-strain responses of TMDs, the bond-forceversus-bond-strain responses are nonlinear (Fig. 4(b)), reaching the maximum strength $(S)$ and then levelling off. Herein, bond stiffness, $K$, is calculated as $K=\lim _{\varepsilon \rightarrow 0} P(\varepsilon) / \mathrm{d} \varepsilon$. Despite their difference in $K$ and $S$, all bonds fracture at approximately the same tensile strain (Table 2). According to our hypothesis, $K / S$ should be lower than 10 for some bonds. However, the values of $K / S$ for all covalent bonds follow the same linear relationship, $S \approx K / 10$, a result consistent with the Griffth's strength limit.

To understand the physical insight behind the nearly identical $K / S$ of all the tested covalent bonds, we analyzed the charge density distribution along these bonds. When covalent bonds form, ${ }^{48-50}$ charge density $\rho(r)$ accumulates along the bond path. The mutual boundary between two atomic volumes intersects this bond path at a saddle point $r_{\mathrm{C}}$ (Fig. 4(c)). The charge density at this point, $\rho\left(r_{\mathrm{C}}\right)$, was found to follow a linear relationship with $K$ (Fig. 4(d)) and $S$ (Fig. 4(e)). This observation is consistent with prior findings that $\rho\left(r_{\mathrm{C}}\right)$ is proportional to the force exerted on the bonding electrons by the nuclei. ${ }^{51}$ For covalent bonds with the same non-metallic elements, their $\rho\left(r_{\mathrm{C}}\right)$, as well as $K$ and $S$, increases as the metallic element goes from the V to VII group. As the group number increases, the transition metal element has more electrons on the first or second shell; both can contribute to the formation of covalent bonds, thereby increasing $\rho\left(r_{\mathrm{C}}\right)$. Given the same metallic element, covalent bonds containing Se atoms have lower $\rho\left(r_{\mathrm{C}}\right)$ than that containing $\mathrm{S}$ atoms, reducing their $K$ and $S$ (Fig. 4(e)). This reduction is because compared to Se atoms, sulfur atoms have higher electronegativity, thereby forming a stronger bond with higher $\rho\left(r_{\mathrm{C}}\right)$. As both $K$ and $S$ follow a linear relationship with $\rho\left(r_{\mathrm{C}}\right)$, the ratio $K / S$ should also increase linearly with $\rho\left(r_{\mathrm{C}}\right)$ (Fig. $4(\mathrm{f})$ ). Nonetheless, such increment is negligible, rising only from 10.2 of $\mathrm{NbSe}_{2}$ to 10.8 of $\mathrm{ReS}_{2}$. Thus, $K / S$ of all the tested covalent bonds can still be treated as a constant. However, compared to the other four TMDs, $\mathrm{ReS}_{2}$ and $\mathrm{ReSe}_{2}$ have stronger covalent bonds but inferior UTS. This inconsistency indicates that the UTS of TMD mainly depends on the topological atomic arrangements rather than their chemcial components.

\subsection{Bond rotation during the deformation of TMDs}

While the covalent bonds in TMDs fracture at a strain of approximately 0.2 (Table 2), the apparent fracture strain of

Table 2 Mechanical properties of the six different covalent bonds in TMDs. $K$ and $S$ are the bond stiffness and bond strength of each bond, respectively

\begin{tabular}{llllll}
\hline & $K(\mathrm{eV})$ & $S(\mathrm{eV})$ & Fracture strain & $K / S$ & $\rho\left(r_{\mathrm{C}}\right)\left(\AA^{-3}\right)$ \\
\hline $\mathrm{MoS}_{2}$ & 115 & 10.93 & 0.21 & 10.52 & 0.072 \\
$\mathrm{MoSe}_{2}$ & 108 & 10.3 & 0.21 & 10.48 & 0.065 \\
$\mathrm{NbS}_{2}$ & 106 & 10.33 & 0.2 & 10.26 & 0.056 \\
$\mathrm{NbSe}_{2}$ & 100 & 9.83 & 0.23 & 10.17 & 0.054 \\
$\mathrm{ReS}_{2}$ & 129 & 11.92 & 0.21 & 10.83 & 0.084 \\
$\mathrm{ReSe}_{2}$ & 120 & 11.33 & 0.2 & 10.59 & 0.078
\end{tabular}

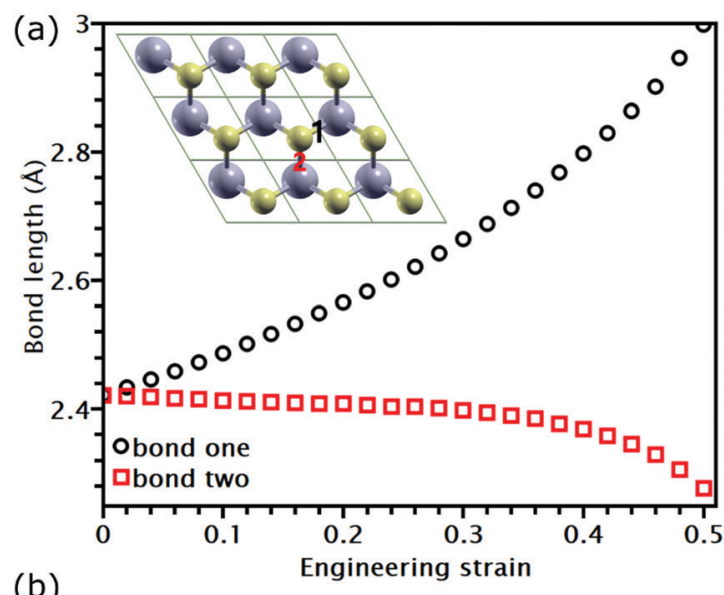

(b)
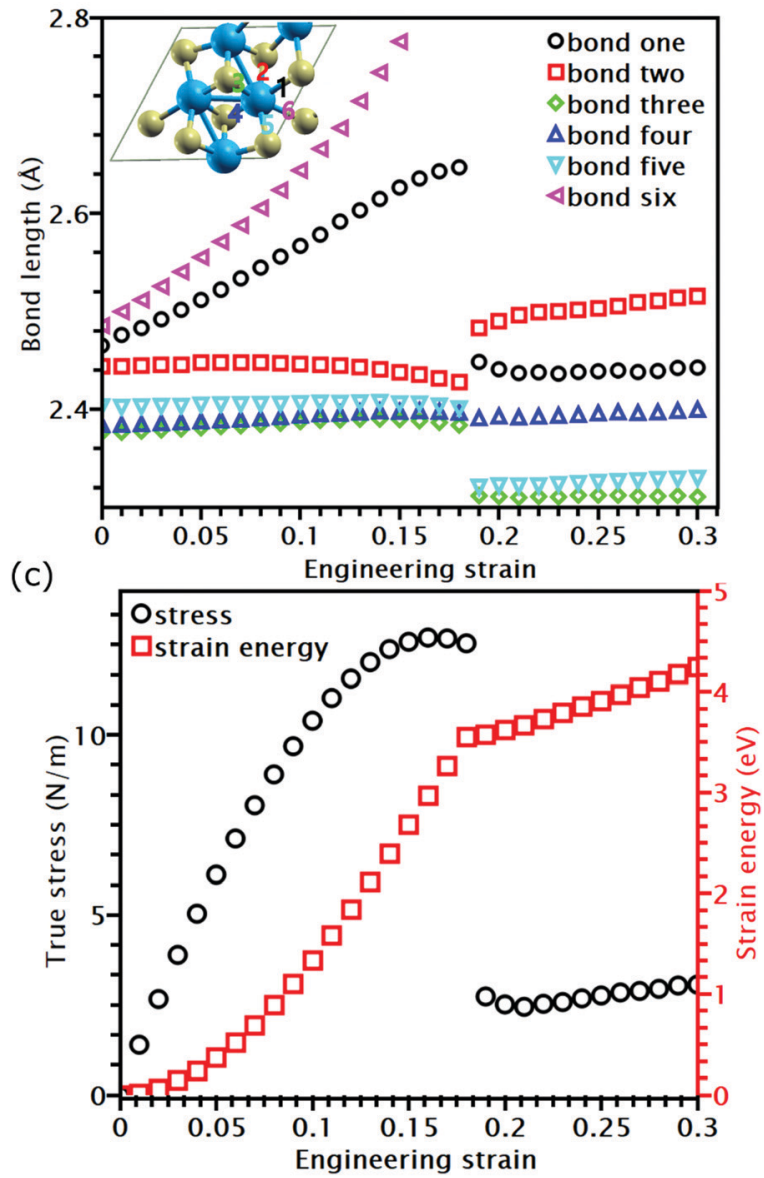

Fig. 5 (a) The evolution of bond length in $\mathrm{MoS}_{2}$. (b) The evolution of bond length in $\mathrm{ReS}_{2}$. (c) The strain-energy increase and the corresponding stress-strain response of $\mathrm{ReS}_{2}$ during $x$-uniaxial tension.

$\mathrm{MoSe}_{2}$ and $\mathrm{MoS}_{2}$ can even reach 0.4 (Table 1). According to previous research, atomic bonds close to the loading direction are more susceptible to fracture. ${ }^{34,35,52}$ However, this mechanism cannot explain why the fracture strain of most TMDs at the UTS point is larger than the bond breaking strain. Furthermore, the Griffth's strength limit predicts that a structure with a high modulus should also have a high ideal strength. This is not the case for $\mathrm{ReS}_{2}$ and $\mathrm{ReSe}_{2}$, which have 
larger moduli than $\mathrm{MoSe}_{2}$ and $\mathrm{MoS}_{2}$ but lower strengths. These phenomena indicate that the failure of TMDs cannot be elucidated only based on bond breaking but should also consider their different topological atomic arrangements.

As shown in Fig. 5(a), $\mathrm{MoS}_{2}$ has only two bond orientations, so half of their bonds are stretched and rotated in uniaxial tension. Since bond rotation also undertakes part of the external deformation, the failure strain can be much larger than the bond fracture strain. However, for $\mathrm{ReS}_{2}$ and $\mathrm{ReSe}_{2}$, their covalent bonds have multiple lengths and directions; therefore, only the bonds lying close to the loading direction and having largest bond lengths are stretched during deformation. For example, as shown in Fig. 5(b), for all the six bonds connected to a Re atom in the unit cell of $\operatorname{ReS}_{2}$, only two of them are elongated, while other bonds are unstretched. Due to the strain-softening effect, the stretched bonds are easier to be further elongated, so the applied deformation is mainly undertaken by the two stretched bonds. Due to such strain concentration, when the longest bond reaches its failure strain, the engineering strain of the overall structure is smaller than the failure strain of the broken bond. After the longest bond is broken, further elongation stretches the remaining bonds. This transition in deformation mechanism results in a non-differentiable point on the energy-strain relation (Fig. 5(c)) and a sudden stress drop of the stress-strain curve. Our results indicate that reducing the variety of bond orientations can increase the failure strain of TMD structures.

\section{Conclusions}

In this work, we performed extensive DFT calculations to evaluate the mechanical properties of six TMDs $\left(\mathrm{MoS}_{2}, \mathrm{MoSe}_{2}\right.$, $\mathrm{NbS}_{2}, \mathrm{NbSe}_{2}, \mathrm{ReS}_{2}, \mathrm{ReSe}_{2}$ ). Our results confirm that the modulus of TMDs increases when the transition metal goes from the V to VII group, or the electronegativity of the nonmetallic atoms increases. However, a high modulus does not guarantee a high ideal strength. In particular, $\mathrm{ReS}_{2}$ and $\mathrm{ReSe}_{2}$ have higher modulus but lower strength than $\mathrm{MoS}_{2}, \mathrm{MoSe}_{2}$ and $\mathrm{NbS}_{2}$, violating the Griffth's strength limit-a linear relationship between the Young's modulus and ideal strength. This violation is not attributed to different chemical components, as our charge-density analysis found that the bond strength $(S)$ and bond stiffness $(K)$ of all the covalent bonds in the six examined TMDs obey a linear relationship with the charge density at the bond saddle point, resulting in $S \approx K / 10$ suitable for all covalent bonds regardless of their chemical components. However, for TMDs having multiple bond orientations, such as $\operatorname{ReS}_{2}$ and $\mathrm{ReSe}_{2}$, different bonds are stretched differently during deformation. Due to the strain-softening effect, these bonds undertaken a larger strain are prone to be further elongated at high strain values. Thus, the deformation is concentrated mainly on these stretched bonds, diminishing the failure strain of the whole structure. Thus, our findings suggest that reducing the variety of bond orientations can increase the failure strain of TMD structures without severe degradation of strength.

\section{Conflicts of interest}

There are no conflicts of interest to declare.

\section{Acknowledgements}

Financial support for this work was also provided through the Natural Sciences and Engineering Research Council of Canada (NSERC), the Ontario Research Fund (ORF), Ontario Graduate Scholarship (OGS), and the Mitacs Accelerate Scholarships. Chandra Veer Singh is an Erwin Edward Hart Endowed Professor of Materials Science \& Engineering-we acknowledge financial support of the Hart fund. The computational resources were provided by the SciNet consortium through the Compute Canada resource allocations.

\section{References}

1 M. S. Jang, H. Kim, Y.-W. Son, H. A. Atwater and W. A. Goddard, Graphene field effect transistor without an energy gap, Proc. Natl. Acad. Sci. U. S. A., 2013, 110, 8786-8789.

2 C.-H. Chang, X. Fan, S.-H. Lin and J.-L. Kuo, Orbital analysis of electronic structure and phonon dispersion in $\mathrm{MoS}_{2}$, $\mathrm{MoSe}_{2}, \mathrm{WS}_{2}$, and WSe $\mathrm{W}_{2}$ monolayers under strain, Phys. Rev. B: Condens. Matter Mater. Phys., 2013, 88, 195420.

3 H. R. Gutiérrez, N. Perea-López, A. L. Elías, A. Berkdemir, B. Wang, R. Lv, F. López-Urías, V. H. Crespi, H. Terrones and M. Terrones, Extraordinary Room-Temperature Photoluminescence in Triangular $\mathrm{WS}_{2}$ Monolayers, Nano Lett., 2013, 13, 3447-3454.

4 K. F. Mak, C. Lee, J. Hone, J. Shan and T. F. Heinz, Atomically Thin $\mathrm{MoS}_{2}$ : A New Direct-Gap Semiconductor, Phys. Rev. Lett., 2010, 105, 136805.

5 B. Radisavljevic, A. Radenovic, J. Brivio, V. Giacometti and A. Kis, Single-layer $\mathrm{MoS}_{2}$ transistors, Nat. Nanotechnol., 2011, 6, 147-150.

6 R. Ganatra and Q. Zhang, Few-Layer $\mathrm{MoS}_{2}$ : A Promising Layered Semiconductor, ACS Nano, 2014, 8, 4074-4099.

7 A. Ramasubramaniam, Large excitonic effects in monolayers of molybdenum and tungsten dichalcogenides, Phys. Rev. B: Condens. Matter Mater. Phys., 2012, 86, 115409.

8 K. F. Mak, K. He, C. Lee, G. H. Lee, J. Hone, T. F. Heinz and J. Shan, Tightly bound trions in monolayer $\mathrm{MoS}_{2}$, Nat. Mater., 2013, 12, 207-211.

9 A. Rawat, N. Jena, D. Dimple and A. De, Sarkar, A comprehensive study on carrier mobility and artificial photosynthetic properties in group VI B transition metal dichalcogenide monolayers, J. Mater. Chem. A, 2018, 6, 8693-8704.

10 O. Lopez-Sanchez, D. Lembke, M. Kayci, A. Radenovic and A. Kis, Ultrasensitive photodetectors based on monolayer $\mathrm{MoS}_{2}$, Nat. Nanotechnol., 2013, 8, 497-501.

11 K. He, C. Poole, K. F. Mak and J. Shan, Experimental Demonstration of Continuous Electronic Structure Tuning via Strain in Atomically Thin $\mathrm{MoS}_{2}$, Nano Lett., 2013, 13, 2931-2936. 
12 A. Castellanos-Gomez, R. van Leeuwen, M. Buscema, H. S. J. van der Zant, G. A. Steele and W. J. Venstra, Single-Layer $\mathrm{MoS}_{2}$ Mechanical Resonators, Adv. Mater., 2013, 25, 6719-6723.

13 C. V. Nguyen, V. V. Ilyasov, H. V. Nguyen and H. N. Nguyen, Band gap and electronic properties of molybdenum disulphide under strain engineering: density functional theory calculations, Mol. Simul., 2017, 43, 86-91.

14 M. G. Sensoy, D. Vinichenko, W. Chen, C. M. Friend and E. Kaxiras, Strain effects on the behavior of isolated and paired sulfur vacancy defects in monolayer $\mathrm{MoS}_{2}$, Phys. Rev. $B$, 2017, 95, 014106.

15 P. Lu, X. Wu, W. Guo and X. C. Zeng, Strain-dependent electronic and magnetic properties of $\mathrm{MoS}_{2}$ monolayer, bilayer, nanoribbons and nanotubes, Phys. Chem. Chem. Phys., 2012, 14, 13035.

16 M. Ghorbani-Asl, S. Borini, A. Kuc and T. Heine, Straindependent modulation of conductivity in single-layer transition-metal dichalcogenides, Phys. Rev. B: Condens. Matter Mater. Phys., 2013, 87, 235434.

17 E. Scalise, M. Houssa, G. Pourtois, V. Afanas'ev and A. Stesmans, Strain-induced semiconductor to metal transition in the two-dimensional honeycomb structure of $\mathrm{MoS}_{2}$, Nano Res., 2012, 5, 43-48.

18 S. Deng, L. Li and M. Li, Stability of direct band gap under mechanical strains for monolayer $\mathrm{MoS}_{2}, \mathrm{MoSe}_{2}, \mathrm{WS}_{2}$ and $\mathrm{WSe}_{2}$, Phys. E, 2018, 101, 44-49.

19 T. Zhu and J. Li, Ultra-strength materials, Prog. Mater. Sci., 2010, 55, 710-757.

20 S. Bertolazzi, J. Brivio and A. Kis, Stretching and Breaking of Ultrathin $\mathrm{MoS}_{2}$, ACS Nano, 2011, 5, 9703-9709.

21 S. Xiong and G. Cao, Molecular dynamics simulations of mechanical properties of monolayer $\mathrm{MoS}_{2}$, Nanotechnology, 2015, 26, 185705.

22 G. Casillas, U. Santiago, H. Barrón, D. Alducin, A. Ponce and M. José-Yacamán, Elasticity of $\mathrm{MoS}_{2}$ Sheets by Mechanical Deformation Observed by in Situ Electron Microscopy, J. Phys. Chem. C, 2015, 119, 710-715.

23 D.-M. Tang, D. G. Kvashnin, S. Najmaei, Y. Bando, K. Kimoto, P. Koskinen, P. M. Ajayan, B. I. Yakobson, P. B. Sorokin, J. Lou and D. Golberg, Nanomechanical cleavage of molybdenum disulphide atomic layers, Nat. Commun., 2014, 5, 3631.

24 J. Zhao, J.-W. Jiang and T. Rabczuk, Temperature-dependent mechanical properties of single-layer molybdenum disulphide: Molecular dynamics nanoindentation simulations, Appl. Phys. Lett., 2013, 103, 231913.

25 J.-W. Jiang, H. S. Park and T. Rabczuk, Molecular dynamics simulations of single-layer molybdenum disulphide (MoS 2): Stillinger-Weber parametrization, mechanical properties, and thermal conductivity, J. Appl. Phys., 2013, 114, 064307.

26 T. Li, Ideal strength and phonon instability in single-layer $\mathrm{MoS}_{2}$, Phys. Rev. B: Condens. Matter Mater. Phys., 2012, 85, 235407.

27 P. Hess, Relationships between the elastic and fracture properties of boronitrene and molybdenum disulfide and those of graphene, Nanotechnology, 2017, 28, 064002.
28 J. Kang, H. Sahin and F. M. Peeters, Mechanical properties of monolayer sulphides: a comparative study between $\mathrm{MoS}_{2}, \mathrm{HfS}_{2}$ and $\mathrm{TiS}_{3}$, Phys. Chem. Chem. Phys., 2015, 17, 27742-27749.

29 P. Hess, Predictive modeling of intrinsic strengths for several groups of chemically related monolayers by a reference model, Phys. Chem. Chem. Phys., 2018, 20, 7604-7611.

30 Y.-J. Zhang, R.-N. Wang, G.-Y. Dong, S.-F. Wang, G.-S. Fu and J.-L. Wang, Mechanical properties of $1 \mathrm{~T}-, 1 \mathrm{~T}^{\prime}-$, and $1 \mathrm{H}-$ MX2 monolayers and their $1 \mathrm{H} / 1 \mathrm{~T}^{\prime}-\mathrm{MX} 2(\mathrm{M}=\mathrm{Mo}, \mathrm{W}$ and $\mathrm{X}=$ S, Se, Te) heterostructures, AIP Adv., 2019, 9, 125208.

31 N. K. Nepal, L. Yu, Q. Yan and A. Ruzsinszky, Firstprinciples study of mechanical and electronic properties of bent monolayer transition metal dichalcogenides, Phys. Rev. Mater., 2019, 3, 073601.

32 W. Shi and Z. Wang, Mechanical and electronic properties of Janus monolayer transition metal dichalcogenides, J. Phys.: Condens. Matter, 2018, 30, 215301.

33 D. M. Guzman and A. Strachan, Role of strain on electronic and mechanical response of semiconducting transitionmetal dichalcogenide monolayers: An ab initio study, J. Appl. Phys., 2014, 115, 243701.

34 H. Sun, S. Mukherjee, Z. Shi and C. V. Singh, Elastomer-like deformation in high-Poisson's-ratio graphene allotropes may allow tensile strengths beyond theoretical cohesive strength limits, Carbon, 2019, 143, 752-761.

35 H. Sun, S. Mukherjee, M. Daly, A. Krishnan, M. H. Karigerasi and C. V. Singh, New insights into the structure-nonlinear mechanical property relations for graphene allotropes, Carbon, 2016, 110, 443-457.

36 P. Giannozzi, S. Baroni, N. Bonini, M. Calandra, R. Car, C. Cavazzoni, D. Ceresoli, G. L. Chiarotti, M. Cococcioni, I. Dabo, A. Dal Corso, S. de Gironcoli, S. Fabris, G. Fratesi, R. Gebauer, U. Gerstmann, C. Gougoussis, A. Kokalj, M. Lazzeri, L. Martin-Samos, N. Marzari, F. Mauri, R. Mazzarello, S. Paolini, A. Pasquarello, L. Paulatto, C. Sbraccia, S. Scandolo, G. Sclauzero, A. P. Seitsonen, A. Smogunov, P. Umari and R. M. Wentzcovitch, QUANTUM ESPRESSO: a modular and open-source software project for quantum simulations of materials, J. Phys.: Condens. Matter, 2009, 21, 395502.

37 J. P. Perdew, K. Burke and M. Ernzerhof, Generalized Gradient Approximation Made Simple, Phys. Rev. Lett., 1996, 77, 3865-3868.

38 H. J. Monkhorst and J. D. Pack, Special points for Brillouinzone integrations, Phys. Rev. B: Solid State, 1976, 13, 5188-5192.

39 J. A. Stewart and D. E. Spearot, Atomistic simulations of nanoindentation on the basal plane of crystalline molybdenum disulfide $\left(\mathrm{MoS}_{2}\right)$, Modell. Simul. Mater. Sci. Eng., 2013, 21, 045003.

40 R. C. Andrew, R. E. Mapasha, A. M. Ukpong and N. Chetty, Mechanical properties of graphene and boronitrene, Phys. Rev. B: Condens. Matter Mater. Phys., 2012, 85, 125428.

41 S. Singh, C. Espejo and A. H. Romero, Structural, electronic, vibrational, and elastic properties of graphene/ $\mathrm{MoS}_{2}$ bilayer heterostructures, Phys. Rev. B, 2018, 98, 155309. 
42 M. K. Mohanta and A. De, Sarkar, Interfacial hybridization of Janus MoSSe and BX (X = P, As) monolayers for ultrathin excitonic solar cells, nanopiezotronics and low-power memory devices, Nanoscale, 2020, 12, 22645-22657.

43 X. Wei, B. Fragneaud, C. A. Marianetti and J. W. Kysar, Nonlinear elastic behavior of graphene: $A b$ initio calculations to continuum description, Phys. Rev. B: Condens. Matter Mater. Phys., 2009, 80, 205407.

44 P. F. Lang and B. C. Smith, Ionization Energies of Atoms and Atomic Ions, J. Chem. Educ., 2003, 80, 938.

45 J.-W. Jiang, Graphene versus MoS2: A short review, Front. Phys., 2015, 10, 287-302.

$46 \mathrm{Z}$. Tu and Z. Ou-Yang, Single-walled and multiwalled carbon nanotubes viewed as elastic tubes with the effective Young's moduli dependent on layer number, Phys. Rev. B: Condens. Matter Mater. Phys., 2002, 65, 233407.
47 E. Cadelano and L. Colombo, Effect of hydrogen coverage on the Young's modulus of graphene, Phys. Rev. B, 2012, 85, 245434.

48 R. F. W. Bader, A quantum theory of molecular structure and its applications, Chem. Rev., 1991, 91, 893-928.

49 R. F. W. Bader, From Schrodinger to atoms in molecules, Pure Appl. Chem., 1988, 60(2), 145-155.

50 R. F. W. Bader and H. Essén, The characterization of atomic interactions, J. Chem. Phys., 1984, 80, 1943-1960.

51 D. Cremer and E. Kraka, A description of the chemical bond in terms of local properties of electron density and energy, Croat. Chem. Acta, 1984, 57(6), 1259-1281.

52 H. Sun, S. Mukherjee and C. V. Singh, Mechanical properties of monolayer penta-graphene and phagraphene: a firstprinciples study, Phys. Chem. Chem. Phys., 2016, 18, 26736-26742. 